\title{
SoxD Transcription Factors: Multifaceted Players of Neural Development
}

\author{
Eun Hye Ji, Jaesang Kim \\ Department of Life Science, Ewha Womans University, Seoul, Korea
}

SoxD transcription factor subfamily includes three members, Sox5, Sox6, and Sox13. Like other Sox genes, they contain the High-Mobility-Group (HMG) box as the DNA binding domain but in addition feature the subgroup-specific leucine zipper motif. SoxD genes are expressed in diverse cell types in multiple organs during embryogenesis and in adulthood. Among the cells expressing them are those present in the developing nervous system including neural stem (or progenitor) cells as well as differentiating neurons and oligodendrocytes. SoxD transcription factors do not contain distinct activator or repressor domain, and they are believed to function in modulation of other transcription factors in promoter-specific manners. This brief review article will attempt to summarize the latest studies on the function of SoxD genes in embryogenesis with a particular emphasis on the regulation of neural development.

Keywords: SoxD, Sox5, Sox6, Sox13, Neural stem cell, Neural development

\section{Introduction to SoxD transcription factors}

SRY (Sex determining Region Y)-Box (Sox) genes encode a group of transcription factors that bind to the minor groove of target DNA elements and regulate transcriptional initiation of nearby promoters (1). Typically numbering in 20 in diverse vertebrate species, the members of this family can be further divided into subgroups based on the level of amino acid conservation within the HMG box and the presence of other motifs (1). Sox5, Sox6, and Sox13 comprise the SoxD subgroup. Aside from the highly conserved HMG box, they feature the leucine zipper motif which allows homo- and hetero-dimerization and con-

\footnotetext{
Accepted for publication April 7, 2016, Published online May 30, 2016 Correspondence to Jaesang Kim

Department of Life Science, Science Bldg C-516, Ewha Womans University, 52 Ewhayeodae-gil, Seodaemun-gu, Seoul 03760, Korea Tel: +82-2-3277-3414, Fax: +82-2-3277-7600

E-mail: jkim1964@ewha.ac.kr

(c) This is an open-access article distributed under the terms of the Creative Commons Attribution Non-Commercial License (http://creativecommons.org/ licenses/by-nc/4.0/), which permits unrestricted non-commercial use, distribution, and reproduction in any medium, provided the original work is properly cited.
}

sequently endows added flexibility in binding site selection (2). SoxD genes are expressed widely during embryogenesis and play critical roles in various aspects of development including cell fate specification (2).

During development, Sox 5 is expressed in subsets of cells in the central nervous system (CNS), cranial ganglia, neural crest and skeletal/cartilage tissues as well as in melanocytes and Th17 cells (2-6). Like Sox 5, Sox6 is expressed in CNS and skeletal/cartilage tissues but in addition is also seen in skeletal and cardiac myocytes and erythroid cells $(2,7)$ Sox13 also is seen in cells in developing CNS and in cartilage progenitors $(2,8)$. Sox13 expression is detected in addition in the developing artery, inner ear, hair follicle and a subset of $\mathrm{T}$ cells $(2,8-10)$.

The best known functions of SoxD genes have to do with cell fate determination and differentiation. Much of the information came from gene inactivation studies in mouse. Sox 5 and Sox6 are particularly well characterized for their redundant and synergistic roles in chondrogenic differentiation (11-14). They enhance the activation of various chondrocyte specific genes by Sox9 (12-14). Loss of either Sox 5 or Sox6 has limited effects but that of both leads to severe chondrodysplasia leading to defects in skeleton formation and embryonic lethality $(11,12)$. Sox 5 is 
also cell-autonomously required for normal Th17 cell differentiation in mouse (6). Independent of Sox5, Sox6 functions in terminal differentiation of cardiac and skeletal muscle cells and erythroid cells $(7,15-17)$. Sox13 is expressed in $\gamma \delta \mathrm{T}$ cells and promotes development of this subpopulation while opposing differentiation of $\alpha \beta \mathrm{T}$ cells (10). Consisting a minority subset of T cells, $\gamma \delta$ T cells express $\gamma$ and $\delta$ receptors rather than $\alpha$ and $\beta$ receptors and show distinct preferred tissues of localization and partly overlapping immune functions with $\alpha$ $\beta$ T cells (10). Sox13-ablated mice show a severe growth retardation compared to wild type mice possibly due to the loss of Wnt signaling modulation for which Sox13 is responsible (10).

\section{Role in cortical neuronal fate specification}

The best characterized role of SoxD genes in neural development has to do with the regulation of neuronal subtype determination. Most of the progress here also is in large part based on analyses of gene-targeted mice during embryogenesis. Sox 5 is expressed in deep cortical layers (layers V, VI, and subplate) from which distinct neuronal populations are born in succession (18). The neurons in the deepest layer, the subplate are born in embryonic day (E) 11.5 while those in layers VI and V show the peaks of generation around E12.5 and E13.5 respectively (18, 19). Neurons in these deep cortical layers, mostly projecting subcortically to spinal cord, pons, midbrain, and thalamus, are born ahead of those in layers I through IV (18). Their layer specification is marked and controlled by presence and/or absence of various transcription factors including Cux1, Cux2, Ferzt2, and Ctip2 (18). Lai and coworkers showed that deletion of Sox 5 results in an abnormal sequence in generation of layer specific neurons and dysregulated gene expression leading to abrogation of layer specific characteristics (19). An independent study by Kwan and coworkers in addition demonstrated that layer $\mathrm{V}$ subcerebral axons are missing and that SP and layer VI axons are misrouted (20). The results indicate that Sox5 is critically involved in multiple aspects of neuronal differentiation for subsets of cortical neurons.

Sox5 and Sox6 show largely mutually exclusive expression patterns during corticogenesis (21). In an elegant study by Azim and coworkers, Sox6 was shown to be expressed in the ventricular zone of pallium (eventually the upper surface of cerebrum) and postmitotic neurons of subpallium (i.e. ganglionic eminence portions of telencephalon) while Sox5 expression was detected in subsets of postmitotic neurons in pallium and ventricular zone of subpallium (21). The complementary expression apparently reflects the mutually repressive function of the two transcription factors at least for the progenitor cells in the ventricular zone (21). Specifically, loss of one gene leads to expansion of the zone expressing the other. Given however that ectopic expression of one transcription factor does not repress the expression of the other, it appears that Sox 5 and Sox6 are necessary but not sufficient for the cross repression. Gene deletion of Sox6 also revealed that Sox6 controls diversity, positioning and maturation of subpallial postmitotic neurons which ultimately constitute cortical interneurons $(21,22)$.

Functional analyses of SoxD proteins in neuronal fate specification outside the cortex are limited. One exception would be the midbrain dopamine (mDA) neurons. Panman and coworkers reported that Sox6 is expressed in a medially located subset of $\mathrm{mDA}$ neurons and its ablation leads to a decrease of substantia nigra pars compacta $\mathrm{mDA}$ neurons and an increase in ventral tegmental area mDA neurons (23). A critical counterpart player appears to be Otx2 which is highly expressed in ventral tegmental area $\mathrm{mDA}$ neurons and deletion of which leads to expansion of the midbrain region occupied by Sox6-positive neurons (23).

Far less is known about the function of Sox13 although a brain-wide expression in the forebrain, midbrain, and hindbrain in post mitotic neurons has been reported (8). More recently, expression of Sox13 has also been shown in oligodendrocyte lineage cells (24). This limited knowledge on Sox13 function likely is due to the redundant role among SoxD genes and a lack of distinct neural phenotypes in Sox13-null mouse model. Therefore, although Sox13 deletion on its own may result in subtle to no visible effect insofar as neuronal fate determination is concerned, combinatorial gene deletion will likely lead to better functional assignments. A careful and detailed comparative analysis of expression pattern for the three genes should be carried out from which testable predictions on function can be made.

\section{Expression and function in neural progenitor cells}

SoxD transcription factors are not just expressed in postmitotic neurons but also in proliferating progenitors in the ventricular and subventricular zones (VZ and SVZ respectively) of the developing nervous system $(21,25-27)$. The expression pattern strongly suggests that they function in determining and/or maintaining 'stemness' of the neural progenitor cells. Alternatively, they may be involved in determining regional identity as their expression 
is seen in more restricted parts of the VZ and SVZ compared to members of the SoxB1 gene subfamily consisting of Sox1, Sox2, and Sox3 (28). These transcription factors show broad and overlapping expression in the $\mathrm{VZ}$ and SVZ of the developing nervous system (28). Consistent with their expression patterns, SoxB1 genes are critical for maintaining 'stemness' of neural progenitors during embryogenesis and into adulthood (29).

In at least two different developmental contexts, SoxD genes have been reported to inhibit differentiation of neural progenitor cells. The first case was oligodendrocyte differentiation in the spinal cord. Using gene targeted models, Stolt and coworkers showed that SoxD proteins control multiple aspects of oligodendrocyte development (27). Specifically, they showed that deletion of either Sox 5 or Sox6 induces premature appearance of cells positive for Sox10 which is a definitive oligodendrocytic lineage marker in the central nervous system. Furthermore, terminal markers of oligodendrocytes such as Proteolipid Protein 1 and Myelin Basic Protein were also precociously expressed. Importantly, they showed that the effect is more pronounced in compound knockout embryos demonstrating the redundant function of the two transcription factors. Recently, by examining Sox6 and Sox13 compound mutation embryo, the Baroti and coworkers demonstrated that Sox13 also contributes to inhibition of premature oligodendrocytic differentiation (24). It should be also noted that migration pattern as well showed anomaly in the gene-targeted embryos consistent with the proposed function of Sox 5 and Sox6 in the multiple stages of oligodendrocyte development (27).

In another study by Lee and coworkers, function of Sox6 in a broader population of neural progenitors was examined (25). The study was based on the finding that Sox6 is a direct regulatory target of Sox2, a critical determinant of 'stemness' of multiple types of stem cells including neural stem cells. Consistent with that Sox6 was a downstream target of Sox2, Sox6 expression was shown to occur among Sox2 positive cells during neural development. Using the chicken in ovo system which had been extensively used to examine spinal cord development, the investigators first demonstrated that Sox 2 induces expression of Sox6 in vivo and subsequently carried out gain- and loss-of-function of Sox6 in the neural progenitors. Remarkably, inhibition of Sox6 expression via RNA interference led to a severe depletion of Sox 2 positive neural progenitors and induction of Isll positive motor neurons in a spatiotemporally precocious manner. Taken together with that ectopic expression of Sox6 resulted in enhanced Sox 2 expression and inhibition of mo- tor neuron differentiation, it was proposed that Sox6 forms a positive feedback network with Sox2 and that together they maintain a pool of neural progenitor or stem cell population (Fig. 1).

Sox 5 shows an expression pattern partially overlapping with that of Sox6 in the VZ and SVZ of developing spinal cord (27). Like Sox6, Sox5 has also been proposed to be a direct regulatory target of Sox2 (25). It is thus possible that Sox 5 plays a similar role for the neural precursors in the developing spinal cord. To date, however, no report has been made in this regard. Rather, a study by Quiroga and coworkers described cell fate specifying function of Sox 5 in dorsal neural progenitors ultimately controlling the specification of dorsal interneurons (26). Whether the function of Sox 5 is restricted to this remains to be further examined.

As described above, Sox5 and Sox6 are also expressed in the VZ's of subpallium and pallium respectively (21). Whether they are directly or indirectly targeted by Sox 2 or other SoxB1 proteins in the cortex and whether they contribute to maintenance of 'stemness' also remain unknown. It has been reported that Sox 5 inhibits premature differentiation of cortical neurons (19). This however is in reference to the proper sequence and timing in the generation of distinct types of cortical neurons and may have little to do with maintenance of 'stemness'. Azim and coworkers, who described the mutually ex-

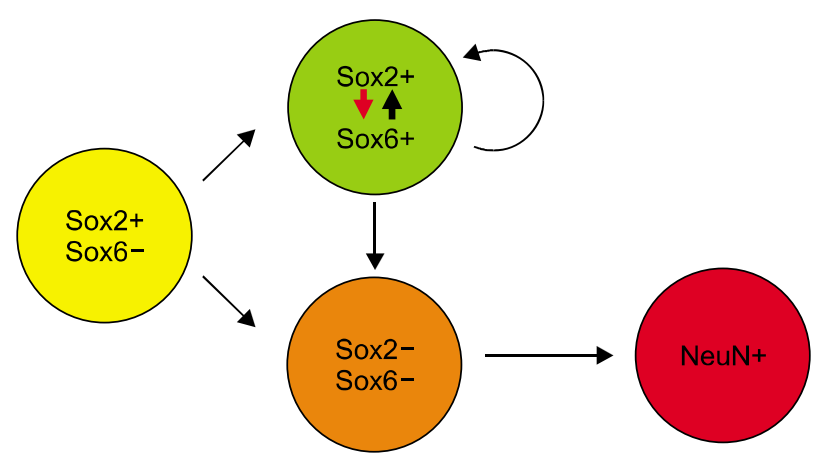

Fig. 1. A model for regulation of neural progenitor cells. Early in development in the absence of neuronal differentiation, progenitor cells are Sox2 (or other SoxB1) single positive. Later on once a subset of progenitor cells undergoes neuronal differentiation, a group of daughter cells expresses other transcription factors (such as Sox6) which form a positive feedback loop with Sox2 and contribute to maintaining the pool of progenitors through the development and into adulthood. Cells that do not become Sox 2 and Sox6 double positive lose Sox2 expression and differentiate into neurons (NeuN positive cell in the figure; $\mathrm{NeuN}$ is a terminal differentiation marker for neurons) or later into oligodendrocytes. Note that the red arrow from Sox 2 to Sox6 implies a direct targeting. 
clusive pattern of expression of Sox 5 and Sox6 also noted that a similar pattern is shown by Ngn2 and Mash1 expressed respectively in pallium and subpallium (21). Interestingly, Sox6 deletion leads to expansion of Mash1 expressing zone into pallium. In addition, other subpallium-restricted genes including Olig2, Dlx1, Dlx2, Gxh2, Ils1 and Meis1 all showed expression in pallium. Loss of Sox 5 however did not show a reciprocal expansion of the zone expressing pallium-specfic genes indicating that regulatory network is not built around Sox 5 and Sox6 as the principal determinants of cell fate. This view is further enhanced by the observation that Sox6-deleted pallial progenitor cells co-express Ngn2 and Mash1 rather than losing Ngn2 expression altogether. Still, it is clear that the two transcription factors contribute to demarcation of distinct zones of differentially fated cells.

\section{Molecular mechanisms of function}

It has been long noted that SoxD transcription factors lack domains associated with transcriptional activation or repression (2). This raises the question as to how they exert their effects as transcription factors. Another issue is how they play apparently distinct roles during multiple developmental stages of given cell lineages. For example, how do their roles switch from maintaining 'stemness' in neural progenitors to regulating timing of differentiation and cell type specific gene expression in developing cortical neurons and oligodendrocytes? While little is known, interactions with other transcription factors and co-factors, both cooperative and inhibitory, are likely key parts of the answer (2).

Sox 5 and Sox6 bind to sites within 'super-enhancers' for the genes involved in chondrocyte development including transcription factors and extracellular matrix (14). Importantly, they promote binding of Sox9 to a distinct but often closely located sites thereby bringing the key activator of chondrogenesis to relevant target genes $(13,14)$. Stunningly, Sox5 and Sox6 inhibit the activity of the same Sox 9 as well as Sox10 during oligodendrocyte development (27). The mechanisms thus may be cell type and target gene specific and include competing for binding sites and for other partner transcription factors such as Olig2 (27). Sox 5 has also been reported to inhibit expression of Sox 10 target genes in the melanocyte lineage by recruiting the co-repressors $\mathrm{CtBP} 2$ and $\mathrm{HDAC} 1$ to their regulatory regions (5). This is in contrast to physical interaction with BMP R-Smads and activation of BMP target genes during early ectoderm patterning and neural crest formation (30). Sox6 also has been reported to interact with HDAC1 and can inhibit $\beta$-catenin dependent transcriptional activation via direct interaction (31). During cortical neuronal differentiation, Sox 5 has been shown to inhibit expression of Fezf2, a gene required for corticospinal neuron identity and connectivity, by competing with Sox 4 and Sox11 for binding sites in an enhancer (32). Finally, Sox13 can block canonical Wnt signaling by directly interacting with TCF1 and promote $\gamma \delta \mathrm{T}$ cell development (10). In sum, SoxD genes can function as either activators or repressors of transcription depending on the developmental and cellular contexts and the nature of cis-regulatory elements of given target genes as well as the presence and interaction with other transcription factors or cofactors. This represents overall a very complex situation that defies generalization, and direct target genes of SoxD transcription factors need to be examined in a gene-by-gene manner.

\section{Conclusion}

The three SoxD genes are expressed in diverse cell types throughout development in partially overlapping manners. Recent years have seen significant advances made in understanding of their roles in neural development. In particular, animal models including knockout mice have made major contributions to demonstrating their functions in maintaining 'stemness' of progenitors, in generation of diverse subtypes of cortical neurons and in properly timed differentiation of oligodendrocytes. Still, our knowledge is extremely limited in terms of specific molecular mechanisms of transcriptional regulation. This is in large part due to the diversity of molecular functions which SoxD transcription factors are capable of performing and the lack of knowledge on direct regulatory target genes in most of the cell types expressing them. This implies that what lies ahead is not a generalization but a gene-by-gene analyses. Specifically, we need to define transcriptional profiles regulated by these genes and determine direct target genes. Modern techniques and tools of molecular biology including RNA-seq, ChIP-seq, and genomic sequences of multiple vertebrate species should enable the first necessary steps to this end.

\section{Acknowledgments}

This study was supported by grants from the Ministry of Health \& Welfare, Republic of Korea (A120262) and from the Ministry of Science, ICT and Future Planning via National Research Foundation via National Research Foundation (2012M3A9B4028766 \& 2013M3C7A1056563). Support also came from the International Research \& Development Program of the National Research Founda- 
tion of Korea (NRF) funded by the Ministry of Science, ICT \& Future Planning (grant No.2015K1A4A3047851).

\section{Potential conflict of interest}

Authors declare that there are no conflicts of interest.

\section{References}

1. She ZY, Yang WX. SOX family transcription factors involved in diverse cellular events during development. Eur J Cell Biol 2015;94:547-563

2. Lefebvre V. The SoxD transcription factors--Sox5, Sox6, and Sox13--are key cell fate modulators. Int J Biochem Cell Biol 2010;42:429-432

3. Morales AV, Perez-Alcala S, Barbas JA. Dynamic Sox5 protein expression during cranial ganglia development. Dev Dyn 2007;236:2702-2707

4. Perez-Alcala S, Nieto MA, Barbas JA. LSox5 regulates RhoB expression in the neural tube and promotes generation of the neural crest. Development 2004;131:44554465

5. Stolt CC, Lommes P, Hillgärtner S, Wegner M. The transcription factor Sox 5 modulates Sox 10 function during melanocyte development. Nucleic Acids Res 2008;36:5427-5440

6. Tanaka S, Suto A, Iwamoto T, Kashiwakuma D, Kagami S, Suzuki K, Takatori H, Tamachi T, Hirose K, Onodera A, Suzuki J, Ohara O, Yamashita M, Nakayama T, Nakajima H. Sox 5 and c-Maf cooperatively induce Th17 cell differentiation via $\operatorname{ROR} \gamma \mathrm{t}$ induction as downstream targets of Stat3. J Exp Med 2014;211:1857-1874

7. Hagiwara N. Sox6, jack of all trades: a versatile regulatory protein in vertebrate development. Dev Dyn 2011;240: 1311-1321

8. Wang Y, Bagheri-Fam S, Harley VR. SOX13 is up-regulated in the developing mouse neuroepithelium and identifies a sub-population of differentiating neurons. Brain Res Dev Brain Res 2005;157:201-208

9. Roose J, Korver W, Oving E, Wilson A, Wagenaar G, Markman M, Lamers W, Clevers H. High expression of the HMG box factor sox-13 in arterial walls during embryonic development. Nucleic Acids Res 1998;26:469-476

10. Melichar HJ, Narayan K, Der SD, Hiraoka Y, Gardiol N, Jeannet G, Held W, Chambers CA, Kang J. Regulation of gammadelta versus alphabeta $\mathrm{T}$ lymphocyte differentiation by the transcription factor SOX13. Science 2007;315:230-233

11. Smits P, Li P, Mandel J, Zhang Z, Deng JM, Behringer $\mathrm{RR}$, de Crombrugghe B, Lefebvre V. The transcription factors L-Sox5 and Sox6 are essential for cartilage formation. Dev Cell 2001;1:277-290

12. Ikeda T, Kawaguchi H, Kamekura S, Ogata N, Mori Y, Nakamura K, Ikegawa S, Chung UI. Distinct roles of Sox5, Sox6, and Sox9 in different stages of chondrogenic differentiation. J Bone Miner Metab 2005;23:337-340

13. Akiyama H, Lefebvre V. Unraveling the transcriptional regulatory machinery in chondrogenesis. J Bone Miner Metab
2011;29:390-395

14. Liu CF, Lefebvre V. The transcription factors SOX9 and SOX5/SOX6 cooperate genome-wide through super-enhancers to drive chondrogenesis. Nucleic Acids Res 2015;43:8183-8203

15. Cantù C, Ierardi R, Alborelli I, Fugazza C, Cassinelli L, Piconese S, BosèF, Ottolenghi S, Ferrari G, Ronchi A. Sox6 enhances erythroid differentiation in human erythroid progenitors. Blood 2011;117:3669-3679

16. Quiat D, Voelker KA, Pei J, Grishin NV, Grange RW, Bassel-Duby R, Olson EN. Concerted regulation of myofiber-specific gene expression and muscle performance by the transcriptional repressor Sox6. Proc Natl Acad Sci U S A 2011;108:10196-10201

17. Cohen-Barak O, Yi Z, Hagiwara N, Monzen K, Komuro I, Brilliant MH. Sox6 regulation of cardiac myocyte development. Nucleic Acids Res 2003;31:5941-5948

18. Leone DP, Srinivasan K, Chen B, Alcamo E, McConnell SK. The determination of projection neuron identity in the developing cerebral cortex. Curr Opin Neurobiol 2008; 18:28-35

19. Lai T, Jabaudon D, Molyneaux BJ, Azim E, Arlotta P, Menezes JR, Macklis JD. SOX5 controls the sequential generation of distinct corticofugal neuron subtypes. Neuron 2008;57:232-247

20. Kwan KY, Lam MM, Krsnik Z, Kawasawa YI, Lefebvre V, Sestan N. SOX5 postmitotically regulates migration, postmigratory differentiation, and projections of subplate and deep-layer neocortical neurons. Proc Natl Acad Sci U S A 2008;105:16021-16026

21. Azim E, Jabaudon D, Fame RM, Macklis JD. SOX6 controls dorsal progenitor identity and interneuron diversity during neocortical development. Nat Neurosci 2009;12: 1238-1247

22. Batista-Brito R, Rossignol E, Hjerling-Leffler J, Denaxa M, Wegner M, Lefebvre V, Pachnis V, Fishell G. The cell-intrinsic requirement of Sox6 for cortical interneuron development. Neuron 2009;63:466-481

23. Panman L, Papathanou M, Laguna A, Oosterveen T, Volakakis N, Acampora D, Kurtsdotter I, Yoshitake T, Kehr J, Joodmardi E, Muhr J, Simeone A, Ericson J, Perlmann T. Sox6 and Otx2 control the specification of substantia nigra and ventral tegmental area dopamine neurons. Cell Rep 2014;8:1018-1025

24. Baroti T, Schillinger A, Wegner M, Claus Stolt C. Sox13 functionally complements the related Sox 5 and Sox6 as important developmental modulators in mouse spinal cord oligodendrocytes. J Neurochem 2015 doi: 10.1111/jnc.13414. [Epub ahead of print]

25. Lee KE, Seo J, Shin J, Ji EH, Roh J, Kim JY, Sun W, Muhr J, Lee S, Kim J. Positive feedback loop between Sox2 and Sox6 inhibits neuronal differentiation in the developing central nervous system. Proc Natl Acad Sci U S A 2014; 111:2794-2799

26. Quiroga AC, Stolt CC, Diez del Corral R, Dimitrov S, Pérez-AlcaláS, Sock E, Barbas JA, Wegner M, Morales AV. 
Sox 5 controls dorsal progenitor and interneuron specification in the spinal cord. Dev Neurobiol 2015;75:522-538

27. Stolt CC, Schlierf A, Lommes P, Hillgärtner S, Werner T, Kosian T, Sock E, Kessaris N, Richardson WD, Lefebvre $\mathrm{V}$, Wegner M. SoxD proteins influence multiple stages of oligodendrocyte development and modulate SoxE protein function. Dev Cell 2006;11:697-709

28. Sarkar A, Hochedlinger K. The sox family of transcription factors: versatile regulators of stem and progenitor cell fate. Cell Stem Cell 2013;12:15-30

29. Bylund M, Andersson E, Novitch BG, Muhr J. Vertebrate neurogenesis is counteracted by Soxl-3 activity. Nat Neurosci 2003;6:1162-1168
30. NordinK, LaBonne C. Sox5 Is a DNA-binding cofactor for BMP R-Smads that directs target specificity during patterning of the early ectoderm. Dev Cell 2014;31:374-382

31. Iguchi $\mathrm{H}$, Urashima $\mathrm{Y}$, Inagaki $\mathrm{Y}$, Ikeda $\mathrm{Y}$, Okamura $M$, Tanaka T, Uchida A, Yamamoto TT, Kodama T, Sakai J. SOX6 suppresses cyclin D1 promoter activity by interacting with beta-catenin and histone deacetylase 1 , and its down-regulation induces pancreatic beta-cell proliferation. J Biol Chem 2007;282:19052-19061

32. Shim S, Kwan KY, Li M, Lefebvre V, Sestan N. Cis-regulatory control of corticospinal system development and evolution. Nature 2012;486:74-79 